\title{
MECHANICAL CHARACTERISTICS OF CVLINDER PIN TUMBLER LOCKS AS THEY RELATE TO RESISTANCE TESTING
}

\begin{abstract}
In recent years, there has been a great development of methodologies, procedures and software tools for quantitative assessment of reliability, efficiency and effectiveness of physical protection systems. Absence of some important input values, as well as missing method to obtain them, is still the most serious problem. This paper is focused on analysis of possibilities how to obtain delay time values in the case of cylinder pin tumbler locks using non-destructive methods of breaking. In the introduction of the article, a proposal of the methodology for testing the mechanical resistance of cylinder pin tumbler locks is elaborated. Consequently, the focus is set on analysis of results obtained during the pilot tests in order to verify the correctness of the proposed methodology. With the tests, it is possible to assess the resulting resistance of cylinder pin tumbler locks also in terms of classification into resistance classes. The pilot testing needs to be implemented mainly due to the lack of a technical standard that processes the issue within the established methodology.
\end{abstract}

Keywords: cylinder pin-tumbler lock, resistance, delay time, methods, testing

\section{Introduction}

The aim of this article is to find out the mechanical resistance of cylinder pin tumbler locks against the non-destructive methods. The first part deals with description and characteristics of cylinder pin tumbler locks; basic terms that are necessary for understanding this field of study; structure of cylindrical pin tumbler locks and the principles of their function. The next section is focused on methods of breaking the cylinder pin tumbler locks, specifically using bumping, picking and racking, which will also be discussed in more detail. The article also includes a proposal for a testing method, which does not have a normative basis for the selected breaking methods. The final part is focused on connecting the results from the tests performed with their implementation in practice.

\section{The cylinder pin tumbler lock - characteristics}

The effort to increase safety brought with it a focus on locking systems for cylinder pin tumbler locks. The principle of their function is based on controlling a latch by a cylinder pin tumbler lock. And it is the cylinder pin tumbler locks which increase the level of passive safety in terms of disallowing entry using unauthorized keys. This type of lock is wide-spread in our territory and it's used in most houses, flats and industrial objects, [1], [2]. The principle of cylinder pin tumbler lock's function is based on inserting a key into the keyhole. Every key is unique in its profile, which ensures the tumblers and driver pins, which are being pressed towards the key using springs, to line up and create a separation between the cylinder and the plug (Figure 1).

As can be seen in Figure 1 left, a common cylinder pintumbler lock is made of several basic parts. The main part is the shell and its shape depends on the use and type of the locking system. Its main function is to connect all parts of the cylinder pin tumbler lock into one compact unit. Plug is the rotating part which can be rotated by $360^{\circ}$ and thus move the locking mechanism. The plug contains in itself all the openings used for inserting the key pins, usually of a cylindrical shape. Their function is to level the surface between the plug and the shell to allow rotation. The key pins can be put into this position only by inserting the correct key. Opposite to the key pins lay the driver pins, which are used to block the plug. Driver pins can be of various shapes, which influence the ability to resist breaking. In case of protecting against bumping, longer driver pins are preferred. Movement of the driver pins is governed by springs, which are placed in the pin changers inside the shell. They serve to block any movement between the shell and the plug of the lock

\footnotetext{
* ${ }^{1}$ Martin Boros, ${ }^{1}$ Anton Siser, ${ }^{2}$ Zoran Kekovic, ${ }^{3}$ Jan Mazal

${ }^{1}$ Faculty of Security Engineering, University of Zilina, Slovakia

${ }^{2}$ Faculty of Security studies, University of Belegrade, Serbia

${ }^{3}$ NATO Modelling \& Simulation Centre of Excellence, University of Defence, Czech Republic

E-mail: martin.boros@fbi.uniza.sk
} 

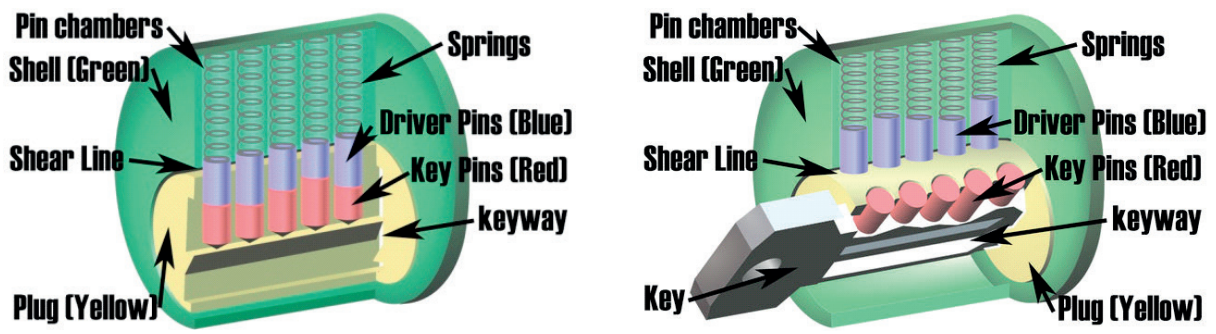

Figure 1 left - Cylinder pin-tumbler lock design and parts, right - rotation of the plug using a key with correct paracentric profile [3]



Figure 2 The various parts and profiles of the key [4]

when no key is inserted. A unique and important characteristic of a cylinder pin tumbler lock is the shape of the keyway inside the plug. It corresponds with the character of the key which consists of several unique edges and profiles (Figure 2). This way, it is possible to ensure opening a cylinder pin tumbler lock with the correct key only.

\section{The cylinder pin-tumbler lock testing using the nondestructive methods}

A common trait of all the passive protection systems is their breachability. This depends on various factors, such as length of the attack, types of tools used and abilities of the perpetrator and the structure of the unit. The breaching itself can be achieved in two ways:

- Destructive method, i.e. using tools or brute force with the aim of breaking the structure and properties of the unit, which usually leads to its destruction or permanent damage;

- Non-destructive method, which uses sophisticated tools, which allow to break a passive protection system once or repeatedly without leaving any visible marks [5].

In this article, the focus is on the latter methods for testing the resistance of cylinder pin tumbler locks. The reason for this is an increase of these methods being used in illegal activities, as well as the insufficient processing of the methodology for testing the delay time property of these elements. The technical standard STN EN 1627 through 1630 focus on resistance in static and dynamic load, as well as a manual breaking attempt. STN EN 1303:2015 only focuses on testing through destructive methods, such as breaking the lock or unscrewing the cylinder pin tumbler locks. However, there is not an existing norm, which would contain the methodology for testing the resistance of a cylinder pin tumbler locks through the non-destructive methods of entry in realistic conditions. The absence of such norm makes it impossible to evaluate the delay time of the mentioned locks without resistance class assignment. Without those values, in turn, one cannot evaluate the level of safety for secured objects where such elements are installed [2].

In relation to cylinder pin tumbler locks, we distinguish three most commonly used methods of breakthrough these locks through non-destructive means:

- Bumping,

- Picking,

- Racking [7].

\subsection{The bumping method}

Bumping represents a dynamic non-destructive method of overcoming a cylinder pin tumbler lock. This method requires the use of a specially modified key. By hitting this key, the energy gets transferred onto the key pins and driver pins, which then jump out of the way and release the plug. The plug can then be rotated and the locking system is deactivated. The whole process begins with preparation, selection or creating of the so-called bump key. The key is inserted into the cylinder pin tumbler lock, its profile and shape must allow for smooth insertion and pulling out of the key. The key is inserted into the keyway stopping before the final key pin. The key is held between two fingers or a specialized tool which allows for greater sensitivity. The second hand holds a screwdriver or a rubber hammer intended for bumping shown in Figure 3. The key is then hit with the rubber part of the screwdriver. When the key is hit, it is slightly rotated. It is necessary to do this at the right moment when the key pins and driver pins are moved away from the shear line - this creates a gap, which allows rotation. The hits and rotation of the key require some sensitivity, skill and training.

When performing the testing, the cylinder pin tumbler locks from security class 1,2 , and 3 underwent the bumping method. In the case of the security class 3 , it was not important to establish a breakthrough time. The test should only verify the claimed resistance against this method. Representatives of the security classes 1 and 2 had the maximum breach time set for 3 minutes and 5 minutes, respectively. For the practical use of the bumping 


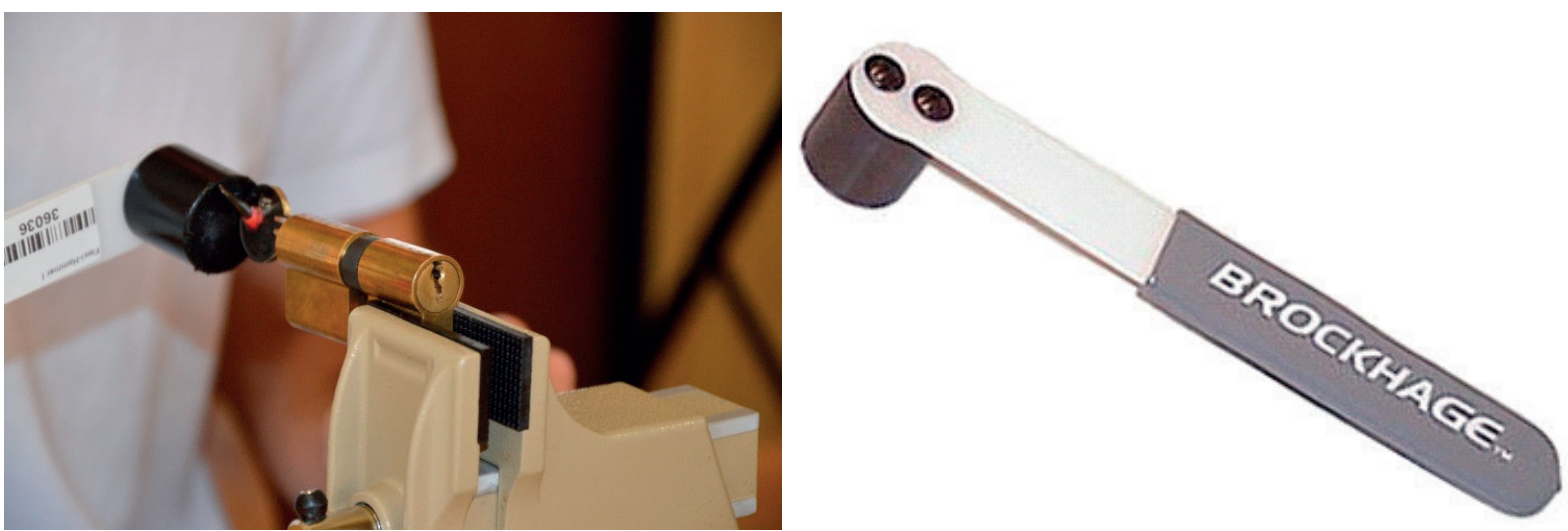

Figure 3 left - Testing cylinder pin-tumbler locks using a bumping method and right-special bump hammer

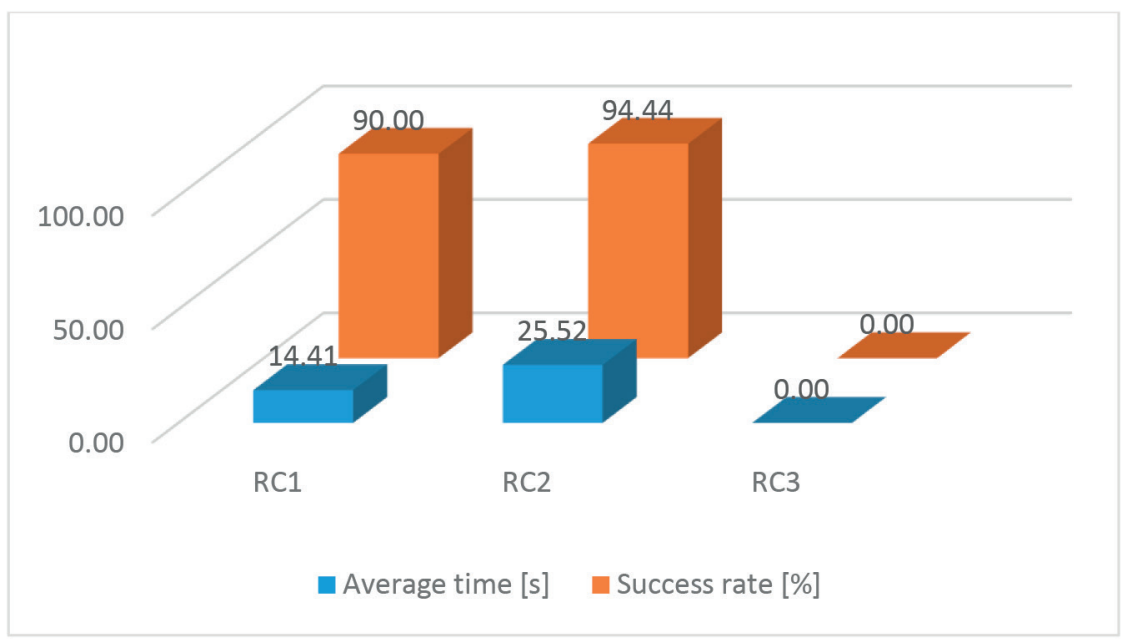

Figure 4 Average times and success rate obtained from testing

method, the experimental units were installed into a vice, which allowed for sufficient grip needed for performing the tests. The bumping method was applied to each unit, measuring the time necessary to break it. The idea was to statistically evaluate the success rate of students in breaking the cylinder pin tumbler lock in a non-destructive way. The testing itself was performed by 3 students, each had 30 tries. The results of the breaking test was recorded in form of numerical results.

When testing the unit that belongs to the security class 1 , the average time necessary for breaking the cylinder pin tumbler lock was calculated to be 14.41 seconds. Out of the 90 tries, 81 were successful and 9 were unsuccessful. The success rate for breaking the lock was $90 \%$. The average breakthrough time in units belonging to security class 2 was 25.52 seconds. Out of the 90 tries, 85 were successful and 5 unsuccessful. The success rate for breaking the lock was $94.44 \%$. In the case of units belonging to security class 3 , the resistance to the bumping method was verified. All acquired results are laid out in Figure 4.

\subsection{The picking method}

The picking method is generally considered as the most difficult way of overcoming the cylinder pin-tumbler locks. It requires a lot of skill, sensitivity and patience from the perpetrator. A potential criminal with sufficient theoretical and practical skills is, however, able to use this method to breach even the complicated security systems. Similar to other methods, the picking method uses imperfections in the factory processing, which allows slight rotation of the plug, as well as leaving it in the right position without using the correct key. Applying tension is the most important stage of the process, which determines the overall success of the operation. The amount of tension causes the driver pins to stay on the edge of the plug and not return back. The entire process has to be done using specialized tension tools shown in Figure 5 left. The second process, occurring simultaneously with continuous application of tension, is pushing individual key pins, which act on the driver pins with the aim of finding the correct unblocked position. This is done using different picks, their shape depending on the type of the cylinder 

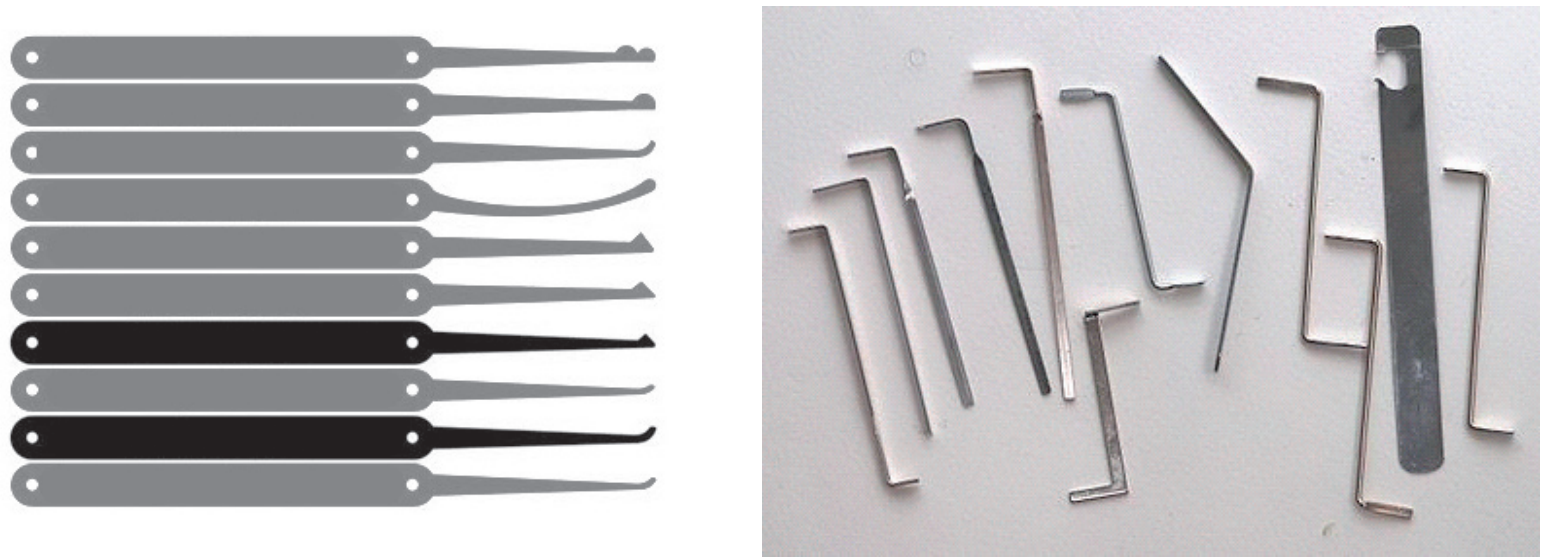

Figure 5 left - Set of various picks and right - tension tools used in lock picking method [8]

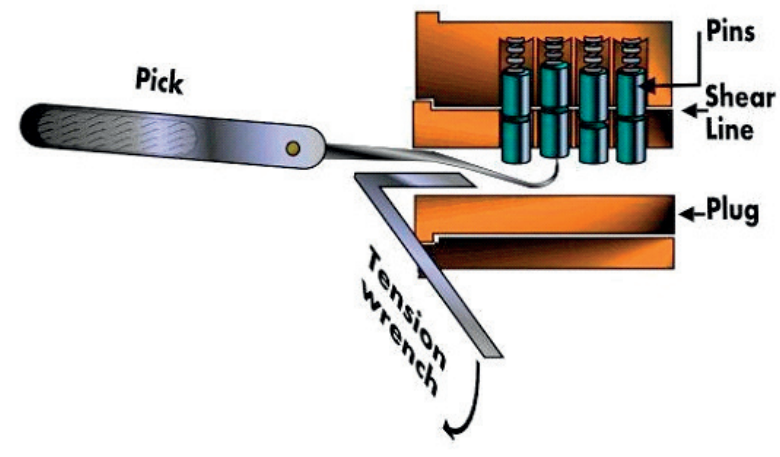

Figure 6 Use of the picking method in practice [9]

pin-tumbler lock. The basic pick types are shown in Figure 5 right. The process of this method is shown inn Figure 6 for easier understanding.

Testing of the picking method was performed on representatives of cylinder pin tumbler locks from resistance class RC1 through RC3 using a process described earlier. In the case of the $\mathrm{RC} 1$ representative, the maximum time for opening the lock was set for 5 minutes. Out of 10 tries to open the lock using the picking method, the lock was breached two times with the average breakthrough time of 4 minutes and 47 seconds. To breach an RC2 cylinder pin tumbler lock, the maximum time was set for 10 minutes. The lock was only overcome once out of 10 tries in 8 minutes and 12 seconds. All other tries were finished after the predetermined amount of time has run out. In the case of the RC3 cylinder pin tumbler lock, the maximum time for overcoming it was set for 15 minutes. Not a single try resulted in opening this lock. The reason for this is the technical solution and quality of the tested unit, which is protected against this method by a blocking driver pin. The result of the testing is shown in Figure 7.

\subsection{The raking method}

Raking is the fastest method of non-destructive breach of a cylinder pin-tumbler lock. The perpetrator does not need very skilled hands, but the method is less effective than the picking method. As with the picking method, there is a very important phase of raking, which is applying the correct tension on the plug. This is done in the same way, using the same tension tools. The important distinction lies in the way the driver pins are pushed by interacting with the key pins. In this method, different picks are used (Figure 8), which are generally longer and have more bends. These picks are inserted into the keyway and by repeated swift movements over the key pins, one is trying to find a position in which the plug can be rotated. The process of this method is shown in Figure 9.

Testing the resistance using the raking method was performed on identical samples as with the case of picking method. The $\mathrm{RC} 1$ representative was breached 18 out of 20 times, average time for opening the lock was 1 minute and 53 seconds. The second tested unit represented the RC2 category resisted only 7 times out of 20 tries. Average breakthrough time was 4 minutes and 13 seconds. Same as with the picking method, the RC3 locks were not broken. The reason for this is the aforementioned technical solution. For an easier understanding of all the results acquired from the testing, all the measured values are projected in graphs in Figure 10.

\section{Conclusions}

The article focused on the problem of testing the mechanical resistance of the cylinder pin tumbler locks. The first part focused on describing the testing of cylinder pin tumbler lock using the non-destructive bumping method. Then the methodology for testing the lock through this method was proposed. Based on the tests performed, one can state that breaching a cylinder pin tumbler lock requires some knowledge and skills, which can be 


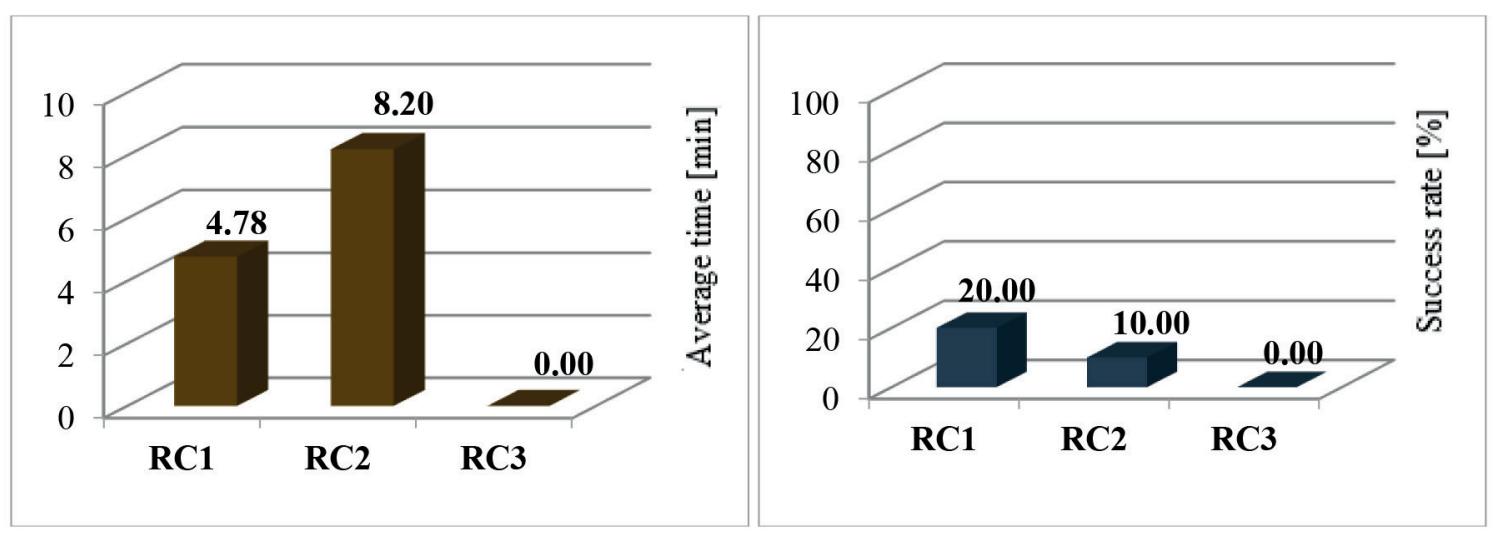

Figure 7 left - Average breakthrough time and right - success rate using the picking method

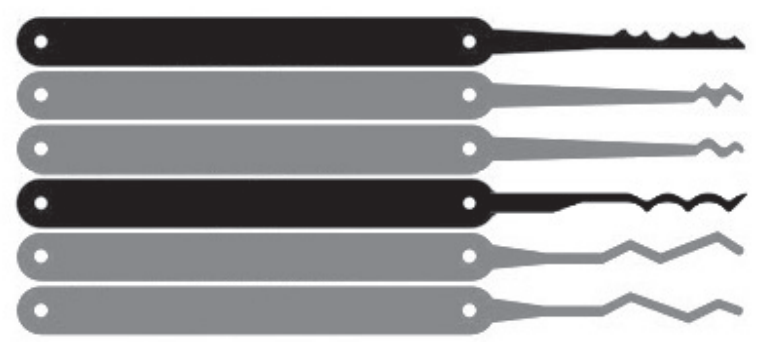

Figure 8 Set of various picks used in the lock-raking method [8]

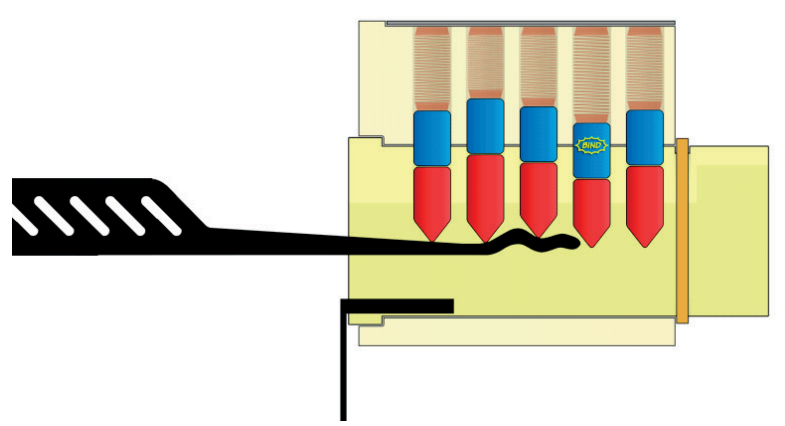

Figure 9 Use of the raking method in practice [9]

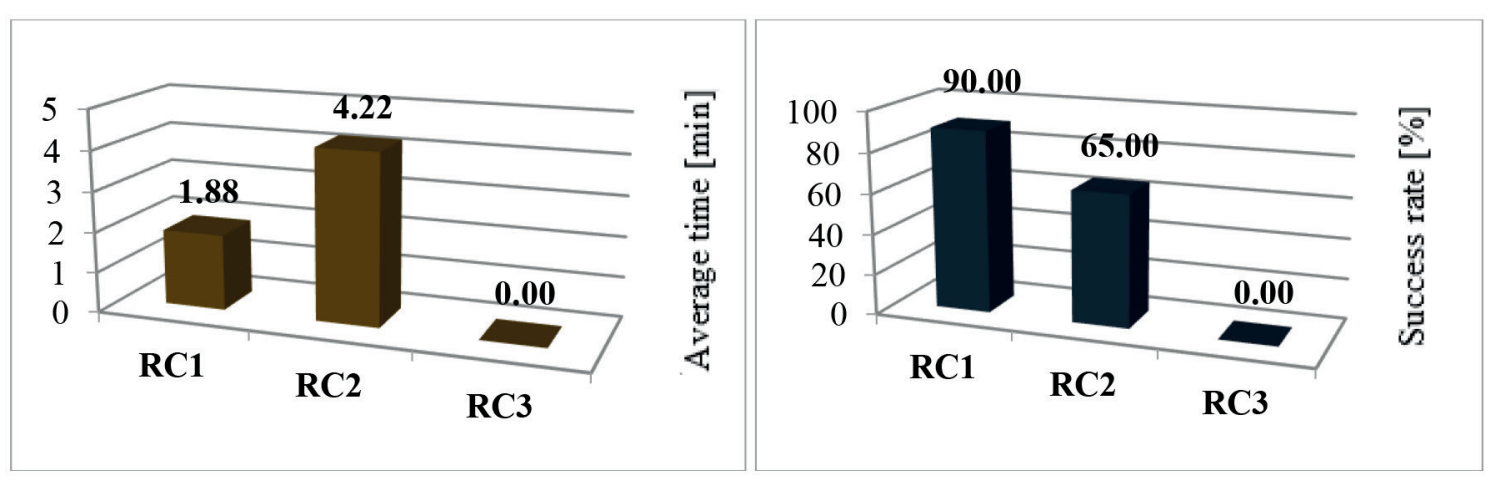

Figure 10 left - Average breakthrough time and right - success rate using the raking method

acquired in a relatively short time; this in turn creates a high level of risk in terms of securing objects. The main benefit of this article was the effort of the authors to point out to an absence of norms, which would regulate the testing of cylinder pin tumbler locks through non-destructive bumping, picking and racking methods and the need to address this situation. At the same time, these tests could be considered a starting point with the aim of creating a basis for further investigation.

\section{Acknowledgments}

This work was supported by the institutional grant project IGP 201701 and IGP 201704. 


\section{References}

[1] MACH, V.: Security Systems - Passive Barriers (in Slovak), first edition. Multiprint, Kosice, p. 199, 2010.

[2] MITRIK, M., MACH, V.: Passive Barriers, first edition. VSBM, Kosice, p. 135, 2008.

[3] How to Pick a Lock (Basics) [online]. Available: www.instructables.com /id/How-to-Pick-a-Lock-Basics/ [accessed: 2018-02-10].

[4] Lock Basics and How to Work with them [online]. Available: www.digitalcitizen.life/book -review-practical-lock-picking-secondedition-deviant-ollam [accessed: 2017-12-22].

[5] IVANKA, J.: Passive Barriers (in Czech), second edition. UTB, Zlin, p. 148, 2014.

[6] LOVECEK, T., REITSPIS, J.: Designing and Assessing Object Protection Systems. EDIS, Zilina, 2011.

[7] GARCIA, M. L.: The Design and Evaluation of Physical Protection Systems. Elsevier, USA, 2001.

[8] Raking vs. Single Pin Picking [online]. Available: https://unitedlocksmith.net/blog/raking-vs-single-pin-picking [accessed: 2018-01-08].

[9] The Rake Method - A Brief Guide [online]. Available: https://www.bumpmylock.com/pages/the-rake-method-a-brief-guide.html [accessed: 2018-01-08]. 